\title{
Stress Monitoring System using Sensors for Drivers
}

\author{
Senthamizh Selvi R', Aishwarya JVR², Deepavarshini $S^{3}$ and Sudha. $S^{4}$ \\ ${ }_{1,2,3,4}$ SRM Easwari Engineering College, Chennai, India
}

\section{ABSTRACT}

The stress level of the driver will have a great impact at the time of driving. This may have impact in driver performance and it may lead to many accidents. Many people tend to lose their lives due to driver's stress level. Something which is not seen and identified is generally defined as stress. In this paper, different sensors like heartbeat, eyeblink sensors are used. Eye blinking, speed, steering angle, turn signal are the parameters that can be monitored while driving. These are generally used to examine the driver's distractions. Real time data collection, sharing of data based on IOT, and data analytics are to be used here to overcome the challenges. In order to predicts the level of stress of the driver and his drowsiness at the rime of driving, heartbeat and eyeblink sensors are used. To use this in real time this sensor is attached to the driver's glass. The data collected using this sensor is passed on to the microcontroller. The real time data is passed on to the system using the RS232 cable. The data collected in real time from COM port is generally obtained using Net beans and these collected data are saved in SQL database. The data that are collected in real time can be tracked by the travel agencies, public care centers, vehicle departure points. The data of the driver is monitored in a system using visual basic. From the system, the driver data is updated into the cloud. If the driver's heartbeat goes abnormal, automatically the engines get slowed down completely. The data collected in real time are refined from Net beans in format of an excel file. These data are then processed to the $\mathrm{R}$ studio for data analytics. In the $\mathrm{R}$ studio, programming is done for both clustering and classification which are processed for abnormal or normal conditions. The final data of the driver which are collected are finally stored in a private cloud for easy access.

\section{KEY WORDS: STRESS DETECTION, DROWSINESS, IOT, DATA ANALYTICS.}

\section{INTRODUCTION}

Human elements are in charge of a significant number of the traffics mishaps out and about. The information introduced in exploration article orders the real hazard factors in charge of traffic mishaps as indicated by their effect as human elements (91\%). Among these, human elements comprise of subjective mistakes (40.7\%), judgment blunders (34.4\%), execution blunders $(10.4 \%)$, and others (16\%). Intellectual blunders show the psychological circumstances where the subjective load,

\section{ARTICLE INFORMATION}

*Corresponding Author: senthamizhselvir74@gmail.com Received 11th Oct 2020 Accepted after revision 29th Dec 2020 Print ISSN: 0974-6455 Online ISSN: 2321-4007 CODEN: BBRCBA

Thomson Reuters ISI Web of Science Clarivate Analytics USA and Crossref Indexed Journal

\section{Clarivate
Analytics}

NAAS Journal Score 2020 (4.31)

A Society of Science and Nature Publication,

Bhopal India 2020. All rights reserved.

Online Contents Available at: http//www.bbrc.in/

Doi: $h t t p: / / d x$.doi.org/10.21786/bbrc/13.13/48 seen by the driver is not normal and moves made also deal with the circumstances in numerous event which is not fitting (Munla et al. 2015). The capacity to identify the advancement of the intellectual load and feelings of anxiety of the driver, it is important to give assistance to the driver to more readily deal with these circumstances (Östlund et al. 2006).

Stress is usually characterized as a sentiment of strain and weight. There is proof that pressure is connected with numerous ailments, assuming a pivotal job in advancement of the cardiovascular illnesses, asthma and this additionally cause fundamental impacts of these illness (Choi and Gutierrez-Osuna 2006). Stress which is identified with a way of life; in this way, particularly for mobile computerized a way of life directing and examination benefits, the need emerges to recognize pressure naturally amid daytime, utilizing physiological information from different sensors. On the off chance that pressure could be dependable what's more, naturally

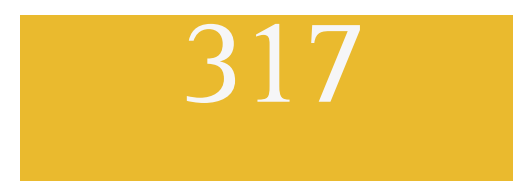


distinguished, this could straightforwardly encourage clients oversee pressure circumstances, and which could likewise be utilized in the medical insight applications (Alexander \& Wang 2017). The methods that are available for the mechanized pressure identification dependent on low cost, pervasive sensors are newer.

Tele monitoring and administration frameworks broaden the skylines of customary social insurance utilizing as it were purpose of care measurement information, however the best possible elucidation furthermore, unwavering quality of the outcomes rely (Wu \& Liu 2017 ) upon the dependability of the estimated information and the sensor itself. A gaming simulator using a real vehicle where the feasibility is also valued. The data of variables which bothers the safety such as distance, behavior are also collected so as to enhance the driver's performance with this simulator (Östlund et al. 2006). Comprising the radio and the related circuit on a single board would be probably bring better outcomes. This is capable of storing the data on a SD card or it could also transmit the information through Bluetooth.

This obtains driver's behavior as ECG, respiration rate, blood flow which is same as how a person will be treated in a hospital. The data that are acquired are completely free from artifacts (Choi \& Gutierrez-Osuna 2009). Physiological data collected in real time may also be useful in determining the stress level of the driver. This information of electrocardiogram, skin effects can tell us the effect of road traffic has high impact on the driver. Many scenarios are analyzed and those conclude that these aspects have effects on driver's (Healey \& Picard 2005). ECG is one of the finest way to check a person's hearts functionality. It is electrical pulse generated and which is viewed in the monitor in graphical form. These plays are a very important role in treatment of cardiac patients as this could probably help them to be aware of their hearts functionality as and when required in a short period and also at a low cost (Price 2010). Driver's drowsiness is one of the most important factor which causes road accidents.

This could probably be stopped, by finding the drivers drowsiness with help of measuring the driver's behavior while driving. By considering vehicle measures, driver's behavioral measures and physiological measures drowsiness of the driver can be determined (Yin et al. 2009). EEG and EMG both together is capable to obtaining the drowsiness of the driver with high accuracy. The signals are separated to various bands and effective transitions are made so as to detect the drowsiness of the driver (Akin et al. 2008). The heart and brain functions together and the data of their behavior is determined in various conditions. These are basically calculated by the analysis of the spectrum and the pattern with synchronizes with the respective frequencies (Kokonozi et al. 2008).

Loss of vigilance is a major reason for road accidents. By monitoring the driver in many aspects like EEG, ECG, EOG during driving helps in reducing the loss of vigilance and being drowsy. The FMIWPT significance has high correlation with the level of drowsiness of the driver (Liang et al. 2009). Blood pressure, heart rate and pal temperature is measured using ANS watch. The activation of this watch may have some abnormal symptoms and balancing the LF, HF are the ways to keep the driver's abnormality in a normal manner (Liang et al. 2009). Fatigue is generally caused due to lack of sleep. The parameters are fixed in order to know the fatigue level of the driver so that measures can be taken to fix them (Yang et al. 2010; Philip et al. 2005; Arun et al. 2020). The remainder of this paper is assembled. Section II provides the stress analysis framework. Section III provides the performance enhancement. Finally, Section IV concludes the paper.

1.2. Methodology used: Driver drowsiness can lead to several accidents and death. It can be detected through different type of sensors. These detection systems are capable of measuring the behavior of the vehicle and the driver which is also helpful in determining the drowsiness of the driver. The real time data collection plays a major role in determining the stress level and drowsiness of the driver from anywhere whenever required. The data are clearly sorted and classified with the data analytics.

Step 1: Accidents due to drowsiness can be controlled and prevented by eye blink sensors using IR rays. This sensor consists of IR transmitter and IR receiver. The transmitter transmits IR ray into the eyes. If the eyes are closed the output will be high whereas if the eye is open, then the output will be low. The output is interfaced with an alarm. This module is connected to the braking system of the vehicle so that it can be used to reduce the speed of the vehicle. If the output is high, then it activated to microcontroller and sets off the alarm. The alarm continues to ring until the driver takes the necessary step to the control of the vehicle. If the driver is unable to take the control of the vehicle, then the microcontroller which is linked to the braking system slows down the vehicle.

Step 2: Stress can also be detected through heartbeat sensor. The heartbeat sensor consists of light emitting diode and detector. The light is emitted through LED, it either reflects or transmits the light. The source of light acts as IR led and detector acts as photodiode. The output is calculated by placing the index finger on the device, it automatically counts the pulses for 30 seconds and get the pulse rate. The heartbeat rate ranges from 68 to 72 . If it falls below 68 or if it is above 72 it is considered as abnormal. This module is connected to the steering angle of the vehicle. The output is interfaced with an alarm. If the output is high, it activates to microcontroller which is linked to the braking system which slows down the vehicle.

Step 3: Arduino is generally said to be a microcontroller which is used to communicate and controlling the operations of several devices. This Arduino consist of fourteen I/O pins. This also consist of a USB port which could be connected to any device. Here microcontroller 
works at mega328, the operating voltage is $5 \mathrm{~V}$, input voltage is $7-12 \mathrm{~V}$, there are about 6 analog input pins, DC current per pin is $40 \mathrm{~mA}$.

Step 4: The data from the driver are collected using Net beans and saved in the SQL database. When any abnormality is detected, alarm automatically rises up and if the driver is unable to take control of the vehicle even after the alarm then the speed of the vehicle is abruptly slowed down. The servlet architecture works with the data base generated and stored. From the data base the servlets program is processed and fed into the http server. From the server through the http protocol the data is made to reach the web browser.

Step 5: Java is a consistent programming language. This is generally capable of giving passive information in areas of probability. In Java once the source code is received, it transfers the data to the Pc for compilation and also gets compiled with the SPARC. From here the data is transmitted to the Java byte code which is a independent platform where the reliability of the data is verified.

Step 6: Data analytics is a process where the raw data is collected in real time. Those data are processed and the datasets are filtered and classified. There is a website which gives a clear interpretation of the drivers driving performance, the skills that he actually carries and also customer is able to view the feedbacks given by the previous customers.

Step 7: From the data collected, number of clusters are formed in order to be processed which is clustered depending on various categories. The values are randomly assigned and the centroid of each cluster is determined. Then the distance of the objects to that of clusters are obtained for further process. The data that are collected from the system initially enters the social media data processing where data are segregated with respect to various aspects depending on the need on which is been classified. This data is further filtered and transferred as error free.

1.3. Proposed system: In this paper, in order to predict the drivers stress level much before the drivers fall sick or gets drowsiness so that so many accidents on road can be avoided. Here two sensors are used as the most important input sources from which three data can be collected in real time. The sensors used are eye blink sensor and heart beat sensor. Heartbeat is clip type one which is attached to the index finger records the heart beat sensor's data in real time. The eyeblink sensor is a glass type one where the sensors detect the drowsiness of the driver and records the data of the driver's drowsiness in real time.

The data collected by the sensor are transmitted to the micro controller. Those data in microcontroller is saved in system using RS232 cable. The data collected from the COM port using Net beans and these data are saved in the SQL database. From the system, the deriver data are updated into the cloud. If the driver heartbeat goes abnormal, the engines are slowed down completely. the data in the net beans are derived as an excel file and these data are generally processed to $\mathrm{R}$ programming studio for data analysis. In the $\mathrm{R}$ programming studio, two main process takes place. They are clustering and classification. The dater in clustering and classification are derived for both normal and abnormal conditions. The daters are finally stored in the cloud which could be accessed easily by anyone at any time.

Figure 1.1: Block diagram - Driver stress monitoring system using sensors

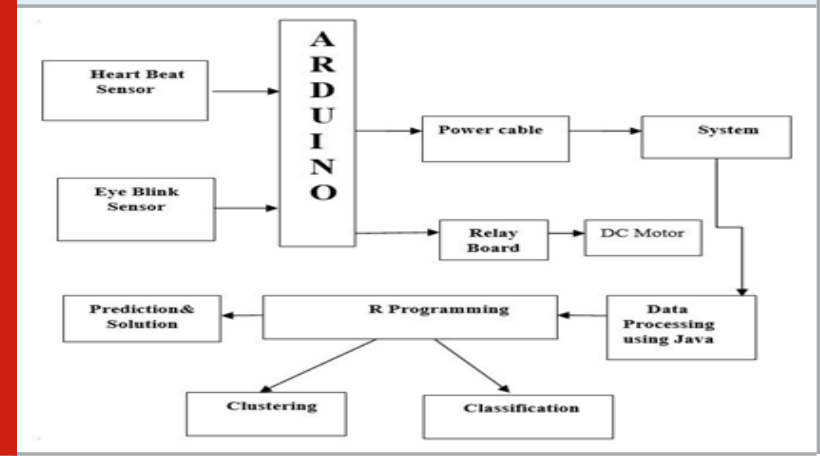

Figure 1.2: Eye blink sensor

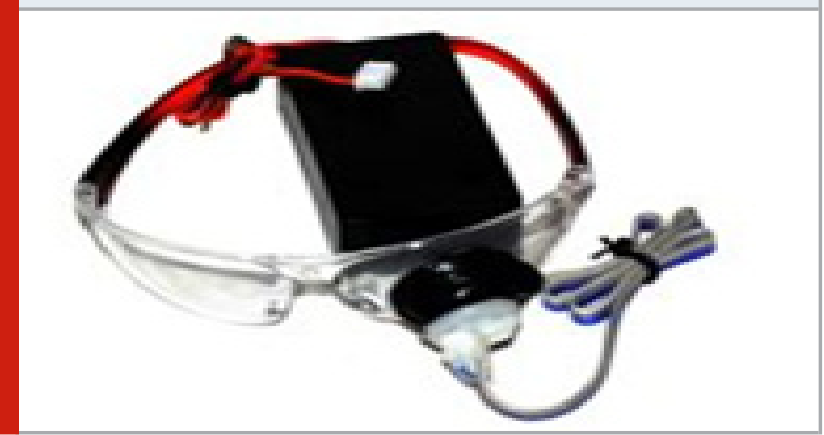

Figure 1.3. Heart beat sensor

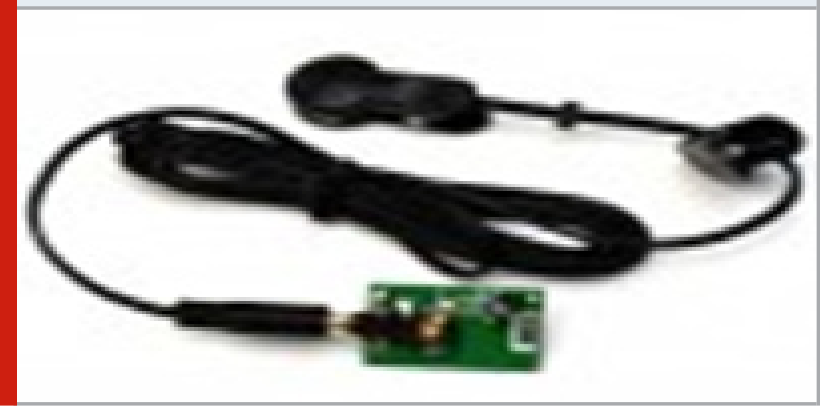

RESULTS AND DISCUSSION

To evaluate the performance of the driver, data stored in SQL database is used. Data from the two sensors such as eyeblink and heartbeat are collected and explored depending on various categories. The motor is completely slowed down as soon as the driver is detected with any of the abnormalities. The data is filtered and processed to the 
$\mathrm{R}$ programming studio where clustering and classification take place to sort the data collected in various respectable aspects. This data from the programming studio is stored in the cloud. One with the driver's identification code and the personal code generated when the user boards the car, or those in the customer serving end could probably be able to access the entire stored data and records of the driver from anywhere and at any time.

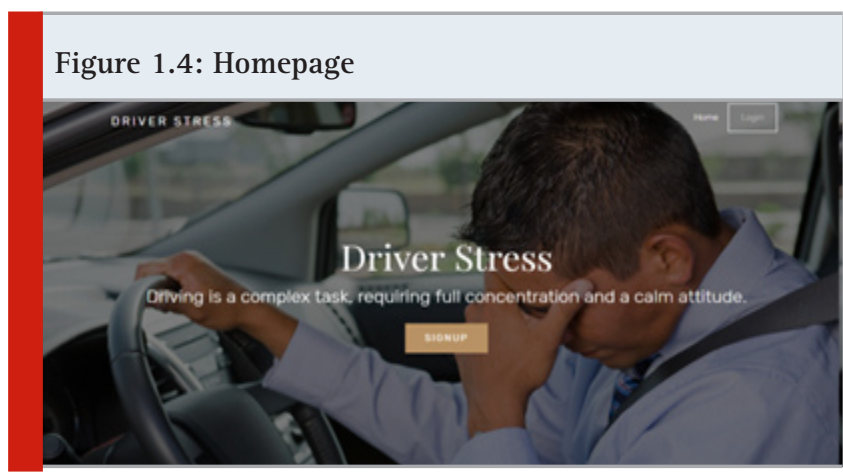

Figure 1.5: Login form

\section{Login Form}

\section{tooin}

Figure 1.6: Registration

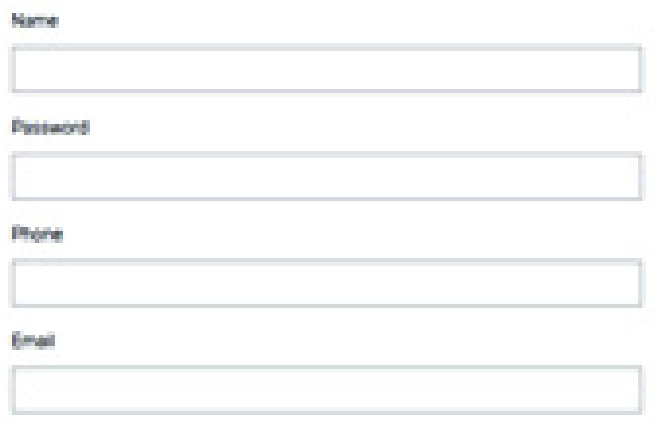

eroistre

In the homepage of the website fig. (1.4), there are two options where an already account user and login and a new user could sign up. In the login form fig. (1.5) the person's name and password generated has to be entered. And if one wants to register fig. (1.6), that person is supposed to enter their name, the generated password, mobile number and their mail address. The live data of the driver is viewed fig. (1.7) which displays the driver's
Figure 1.7: Live data

\section{Check Your Live Data}

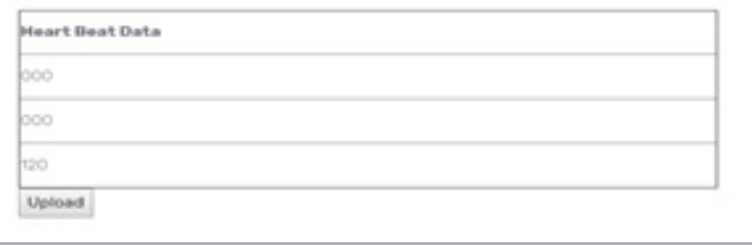

Figure 1.8: User details

\section{Check Your Details}
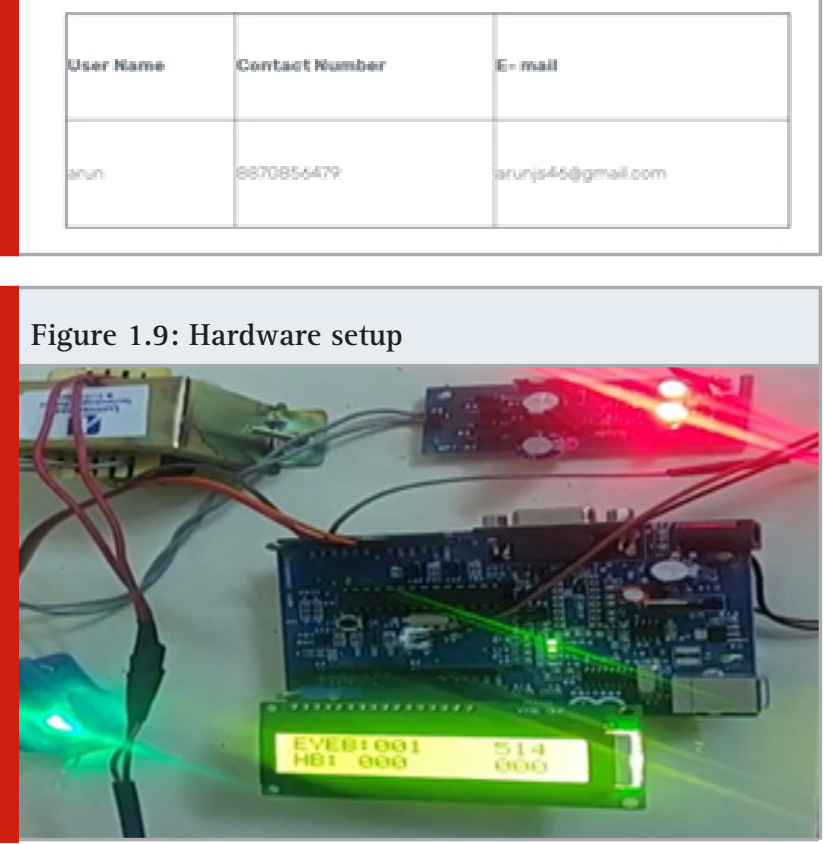

heartbeat of every second in real time. This website gives a clear interpretation of the drivers driving performance, the skills that he actually carries and also customer could view the feedbacks given by the previous rides that he has taken

1.4.1. Data analytics: Data analytics is general process where the raw data collected in real time is combined and sorted. Those data are processed and the datasets are filtered and classified. From the data derived, algorithms and models are also formed in order to generate reports with gradual increase or decrease in the behavioral characteristics of the driver. The entire data is collected and is formed as a product where in it is absolutely useful in future to view the performance of the data whenever and where ever required.

1.4.2. Clustering: $K$ means clustering is generally a learning algorithm which is used to cluster various data collected and analyzed by the programming studios. From the data collected, number of clusters are formed in order to be processed which is clustered depending 
on various categories. The values are randomly assigned and the centroid of each cluster is determined. Then the distance of the objects to that of clusters are obtained for further process. Later, the grouping of the data obtained is completely based on the minimum distance to the maximum distance (Arun et al. 2020). If the random number in the earlier stage fails to find the centroid of any cluster those data are moved to separate group where there are no objects present and also they are filtered out of the entire clustering process.

Figure 1.10: Net beans platform

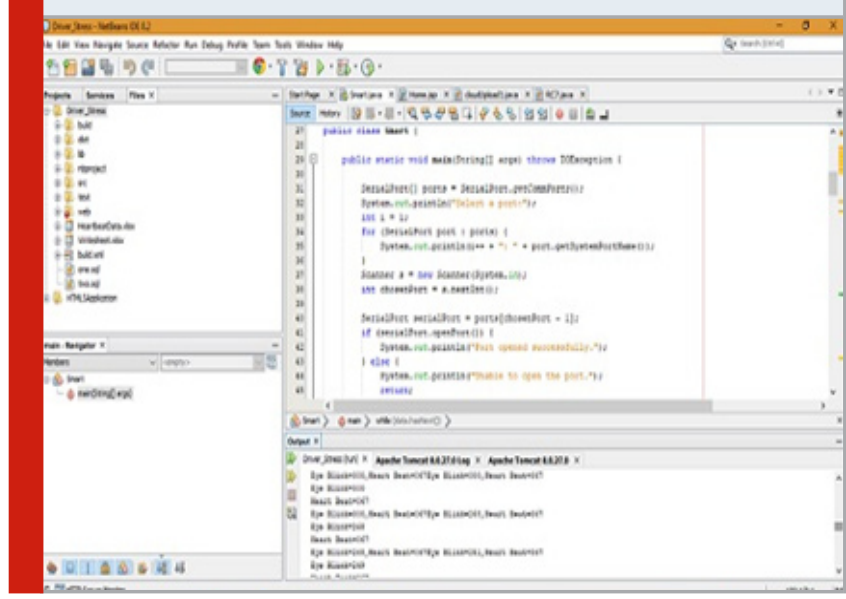

Figure 1.11: SQL Database

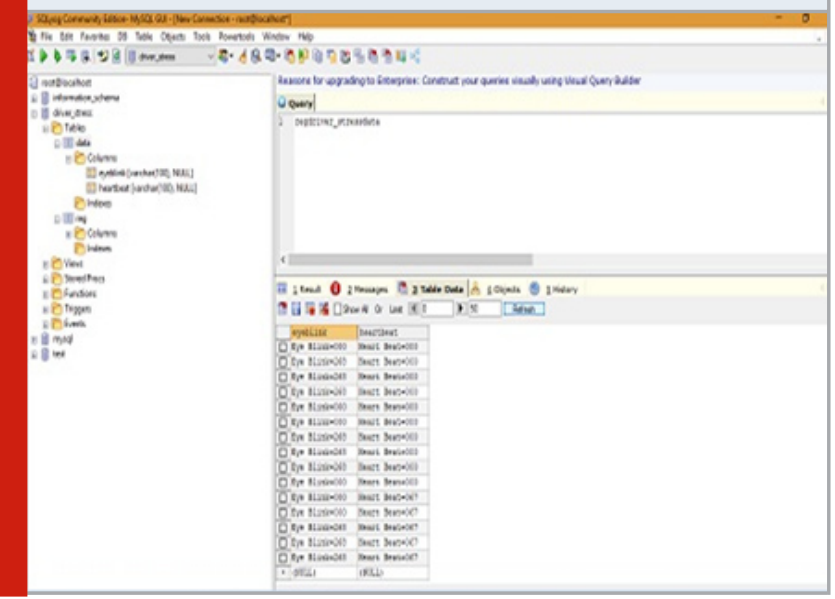

Figure 1.12: Data collected in cloud

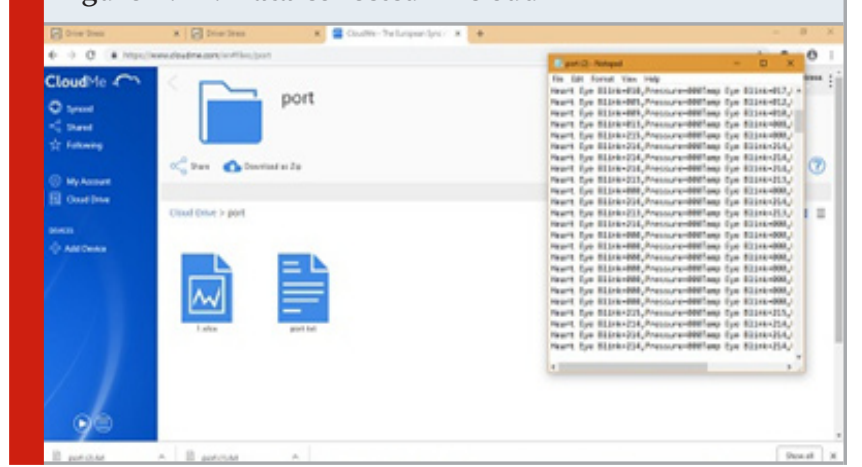

Figure 1.13: Data sharing

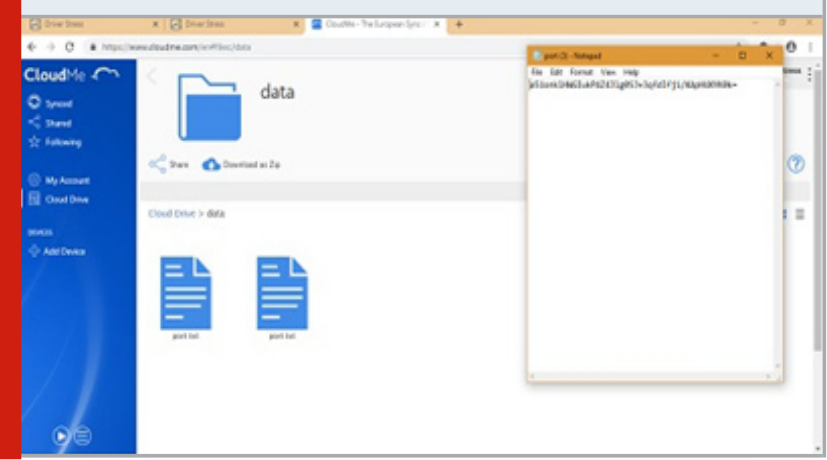

1.4.3. Classification: The naïve bayes classification is the most logical way of classification available in the data analytics processes available. In this type of classification, the data that are collected from the system initially enters the social media data processing where data are segregated with respect to various aspects depending on the need on which is been classified. This data is further filtered and transferred as error free. From the data filtered the extraction of the sentiment is done and the other network also combine to give a proper input to the machine learning algorithms. In the classifier, the data is completely filtered and the analyzation of data is completed. After this analyzation, the data obtained are classified depending on the various categories which are assigned to be needed.

\section{CONCLUSION}

This paper proposes a stress monitoring system based on physiological signals. It describes the behavior of the driver under normal and abnormal situations. It provides the real time information on the state of mind of an individual. The database collected from the driver is generally stored in cloud. These data can be viewed by the customers as well as the travel agencies. With the help of this stress monitoring system, the stress level of the driver can be detected such that the accidents could be prevented.

Moreover, their characteristics in terms of computational cost and hardware requirements make the stress detection system to be embedded in order to increase the overall security. As far as the future work is concerned, implementation of the proposed system on hardware devices and the application of this system in mobile devices is recommended. This paper concludes that, stress level of the driver has severe impact on the road accidents which is very commonly happening, the only way to restrict this is that monitoring the driver's performance and taking necessary actions much before the accidents occur and the same could be achieved with the proposed methodologies.

\section{REFERENCES}

Akin M., Kurt M.B., Sezgin N., and Bayram M. (2008) Estimating vigilance level by using EEG and EMG signals. Neural Computing and Applications, vol. 17, 
pp. 227-236.

Alexander C.A., and Wang L. (2017) Big data analytics in heart attack prediction. J Nurs Care, vol. 6, pp. 21671168, .

Aravind K. (2017) Automation of space management in vehicle parking using PLC and SCADA. International Journal of MC Square Scientific Research, vol. 9, pp. 135-144, .

Arun, M., Baraneetharan, E., Kanchana, A. and Prabu, S., (2020). Detection and monitoring of the asymptotic COVID-19 patients using IoT devices and sensors. International Journal of Pervasive Computing and Communications.

Bhoi A. K., Mallick P. K., Liu C. M., \&t Balas V. E. (2021) Bio-inspired Neurocomputing, Springer Nature.

Choi J., and Gutierrez-Osuna R. (2009) Using heart rate monitors to detect mental stress. In 2009 Sixth International Workshop on Wearable and Implantable Body Sensor Networks, pp. 219-223.

Healey J.A., and Picard R.W. (2005) Detecting stress during real-world driving tasks using physiological sensors. IEEE Trans. Intell. Transp. Syst, vol. 6, pp. 156-166.

Kokonozi A.K., Michail E.M., Chouvarda I.C., and Maglaveras N.M. (2008) A study of heart rate and brain system complexity and their interaction in sleepdeprived subjects. In 2008 Computers in Cardiology, pp. 969-971.

Liang W.C., Yuan J., Sun D.C., and Lin M.H. (2009) Changes in physiological parameters induced by indoor simulated driving: Effect of lower body exercise at midterm break. Sensors, vol. 9, pp. 6913-6933.

Mallick P. K., Balas V. E., Bhoi A. K., and Chae G.-S. (Eds.) (2020) Cognitive Informatics and Soft Computing: Proceeding of CISC 2019, Vol. 768.
Mallick P. K., Balas V. E., Bhoi A. K., and Zobaa A. F. (Eds.) (2019) Cognitive Informatics and Soft Computing: Proceeding of CISC 2017, Vol. 768.

Mishra S., Tripathy H. K., Mallick P. K., Bhoi A. K., and Barsocchi P. (2020) EAGA-MLP-An Enhanced and Adaptive Hybrid Classification Model for Diabetes Diagnosis. Sensors, vol. 20, pp. 4036.

Munla N., Khalil M., Shahin A., and Mourad A. (2015) Driver stress level detection using HRV analysis. In 2015 international conference on advances in biomedical engineering (ICABME), vol. 61-64.

Östlund J., Nilsson L., Törnros J., and Forsman A. (2006) Effects of cognitive and visual load in real and simulated driving. Statens väg-och transportforskningsinstitut. Philip P., Sagaspe P., Moore N., Taillard J., Charles A., Guilleminault C., and Bioulac B.: Fatigue, (2005) sleep restriction and driving performance. Accident Analysis Et Prevention, vol. 37, pp. 473-478.

Price D. (2010) How to read an electrocardiogram (ECG). Part 1: Basic principles of the ECG. The normal ECG. South Sudan Medical Journal, vol. 3, pp. 26-31.

Ren Z., Qian K., Wang Y., Zhang Z., Pandit V., Baird A., and Schuller B. (2018) Deep scalogram representations for acoustic scene classification. IEEE/CAA Journal of Automatica Sinica, vol. 5, pp. 662-669.

Wu C., and Liu Y. (2017) Queuing network modeling of driver workload and performance. IEEE Trans. Intell. Transp. Syst, vol. 8, pp. 528-537.

Yang G., Lin Y., and Bhattacharya P. (2010) A driver fatigue recognition model based on information fusion and dynamic Bayesian network. Information Sciences, vol. 180, pp. 1942-1954.

Yin B.C., Fan X., and Sun Y.F. (2009) Multiscale dynamic features based driver fatigue detection. Int. J. Pattern Recognit Artif Intell, vol. 23, pp. 575-589. 